\title{
Venous Thromboembolism in COVID-19: Towards an Ideal Approach to Thromboprophylaxis, Screening, and Treatment
}

\author{
Madan Raj Aryal ${ }^{1} \cdot$ Rohit Gosain ${ }^{1}$ - Anthony Donato ${ }^{2} \cdot$ Ranjan Pathak $^{3}$ - Vijaya Raj Bhatt ${ }^{4} \cdot$ Anjan Katel $^{5}$. \\ Peter Kouides ${ }^{6}$
}

Published online: 11 June 2020

(C) Springer Science+Business Media, LLC, part of Springer Nature 2020

\begin{abstract}
Purpose of Review Novel coronavirus disease 2019 (COVID-19) has been associated with an increased risk of arterial and venous thromboembolic (VTE) diseases. However, there is a limited amount of data regarding the prevention and management of VTE in severe hospitalized COVID-19 patients.

Recent Findings In this article, we review currently available clinical data, and mechanisms for COVID-associated coagulopathy, and propose algorithms for screening, prevention (including extended-duration prophylaxis), and treatment of these patients. Summary Although these recommendations are subject to change given rapidly evolving data, we provide a framework that can guide clinicians in managing thrombotic complications in this challenging condition.
\end{abstract}

Keywords COVID-19 • Venous thromboembolism · Thromboprophylaxis · Deep vein thrombosis · Pulmonary embolism · Extended prophylaxis

\section{Introduction}

Studies have previously shown that both pneumococcal and influenza infections can be associated with increased risks of venous thromboembolism (VTE) [1, 2]. In critically ill influenza A $\mathrm{H} 1 \mathrm{~N} 1$ patients with acute respiratory distress syndrome (ARDS), empiric systemic anticoagulation was associated with decreased rates of VTE [2]. Similarly, novel coronavirus disease 2019
(COVID-19) has been thought to predispose to both venous and arterial thromboembolic diseases. Prevalence can be as high as $25 \%$ in patients that develop ARDS and can lead to higher rates of complications and poor overall prognosis [3].

Given the lack of clear guideline recommendations on the prevention and management of VTE in severe hospitalized COVID-19 patients, we believe that the following clinical questions deserve further study and clarification.

\author{
Madan Raj Aryal \\ madan.aryal@ roswellpark.org \\ Rohit Gosain \\ Rohit.gosain@roswellpark.org \\ Anthony Donato \\ Anthony.donato@towerhealth.org \\ Ranjan Pathak \\ Ranjan.Pathak@yale.edu \\ Vijaya Raj Bhatt \\ vrbhatta@gmail.com \\ Anjan Katel \\ anjan.katel3@gmail.com \\ Peter Kouides \\ Peter.Kouides@rochesterregional.org
}

1 Department of Medicine, Roswell Park Comprehensive Cancer Center, University at Buffalo, Elm and Carlton Streets, Buffalo, NY 14263, USA

2 Department of Medicine, Tower Health System, 6th Avenue and Spruce Street, West Reading, PA 19611, USA

3 Department of Medicine (Section of Medical Oncology/ Hematology), Yale University School of Medicine, 333 Cedar Street, Room WW201, New Haven, CT 06520, USA

4 Section of Hematology, University of Nebraska, 42nd and Emile, Omaha, NE 68198, USA

5 Department of Medicine, School of Medical Sciences, Kathmandu University, Dhulikhel, Nepal

6 Department of Medicine, Rochester Regional Health, University of Rochester, 100 Kings Highway South, Rochester, NY 14617, USA 


\section{Is There a Biologic Basis for Increased Risk of VTE in COVID-19?}

Increased VTE events in COVID-19 are thought to be due to immobilization, excessive inflammation, and diffuse intravascular coagulation (DIC) [4]. Although not primarily a thrombotic process, inflammation and hypoxia with acute lung injury leads to a profound inflammatory state due to cytokine storm, macrophage, and endothelial activation-related processes associated with a surge in IL-1, IL-6, IL-8, and TNF-alpha which suggest that there are biological evidences for the thrombotic process. Evidence of coagulopathy has been reported, with patients demonstrating often markedly elevated serum levels of D-dimer, lactate dehydrogenase, and total bilirubin with slight prolongation or no changes in partial thromboplastin time (PT) or activated partial thromboplastin time (PTT) [5]. Diffuse microvascular thrombi with possible thrombotic microangiopathy in multiple organs have been reported on autopsy review without viral infiltrates [6]. In addition, the association of COVID-19 with clinically significant coagulopathies, multiple infarcts, and antiphospholipid antibodies has also been described [7]. However, the association between COVID-19 and antiphospholipid syndrome (APS) remains speculative at this point given that the definitive diagnosis of APS requires persistence of IgG antibodies (rather than IgA antibodies as reported) at 12 weeks along with thrombotic events meeting the Sapporo criteria. In patients that harbor rare germline mutations in complement regulatory genes, complement activation can lead to antiphospholipid antibody-induced thrombotic events [8], suggesting a possible role for complement blockade in managing complement-mediated APS [6].

\section{Should We Screen all Hospitalized Severe COVID-19 Patients for VTE?}

Although the incidence of VTE seems to be higher in COVID-19
patients, further studies on VTE in these patients are needed.
Confirmation of such a relatively high rate of VTE would war-
rant consideration for screening lower limb ultrasounds and con-
sideration of intermediate to full-dose anticoagulation akin to the
approach used in heparin-induced thrombocytopenia without
thrombosis. Based on the current evidence, International
Society on Thrombosis and Hemostasis (ISTH) recommends
measuring D-dimer, PT, PTT, and platelet count in all hospitalized
patients with COVID-19 [9]. Rapid deterioration in oxygen sat-
uration or increased dead space ventilation might be better indi-
cators of a new VTE event, rather than relying solely on hema-
tological abnormalities. Given logistical issues resulting from the
strict isolation in COVID-19 patients, it is likely that there is a
higher threshold to perform diagnostic imaging in these patients.
Many critical care units in high-income countries utilize point-of-
care ultrasound, which may be utilized for screening purposes.
The use of dedicated ultrasound for COVID-19-infected patients may limit the risk of cross-contamination to patients without COVID-19. Elevations in D-dimer are very common in this group and are not specific for VTE events [5].

Klok et al. evaluated the incidence of the composite outcomes of VTE and arterial thrombotic complications in all COVID-19 patients admitted to the intensive care unit (ICU) [4]. A total of 184 consecutive patients with COVID-19 pneumonia admitted to the ICU were evaluated. All patients received at least standard-dose thromboprophylaxis. Among these, only those patients with a clinical suspicion for VTE underwent diagnostic evaluation with further imaging. Confirmed VTE was noted in $27 \%$ and arterial thrombotic events in $3.7 \%$ of patients. Pulmonary embolism (PE) was the most frequent VTE (81\%). Spontaneous prolongation of the PT by more than $3 \mathrm{~s}$ or PTT by more than $5 \mathrm{~s}$ was an independent predictor of thrombotic complications. Similarly, Tang et al. reported an association between 28-day mortality with D-dimer, PT, age, and platelets on multivariate analyses [10]. This study was limited due to its retrospective design and a much lower rate of thromboprophylaxis compared with Western countries (only a fifth of patients were administered thromboprophylaxis) (Table 1).

\section{What Is the Appropriate VTE Prophylaxis and Treatment for Severe COVID-19 Patients?}

Given the high risk of VTE in critically ill COVID-19 patients, appropriate VTE prophylaxis seems to be an important part of managing these patients. Many critically ill patients we considered in our observational studies have high Padua scores and are associated with lower mortality rates when given LMWH or heparin; we suggest that all severely ill COVID-19 patients undergo weight-based thromboprophylaxis. While the use of standard-dose thromboprophylaxis in hospitalized patients is acceptable, the high reported incidence of VTE (25-31\%) suggests that higher doses, i.e., enoxaparin $0.5 \mathrm{mg} / \mathrm{kg}$ twice daily, may be more appropriate $[3,4]$. Given that thrombocytopenia in COVID19 patients may be less profound than that in other sepsis syndromes [11], prophylactic anticoagulation is likely to be feasible.

Anticoagulation treatment in severe COVID-19 patients seems to be associated with better outcomes [10]; empiric anticoagulation should be strongly considered in patients with high suspicion for VTE but cannot undergo diagnostic imaging, in the absence of contraindications to anticoagulation therapy. Patients with physical findings consistent with superficial or DVT should also undergo therapeutic anticoagulation. Helms et al. found that despite prophylactic anticoagulation, a high number of patients with COVID-19 ARDS developed lifethreatening thrombotic complications; hence, higher anticoagulation targets have been suggested (Fig. 1) [12].

A large study of 1087 patients found that nearly $40 \%$ had high-risk Padua scores (defined as score of $\geq 4$ ), and that higher 


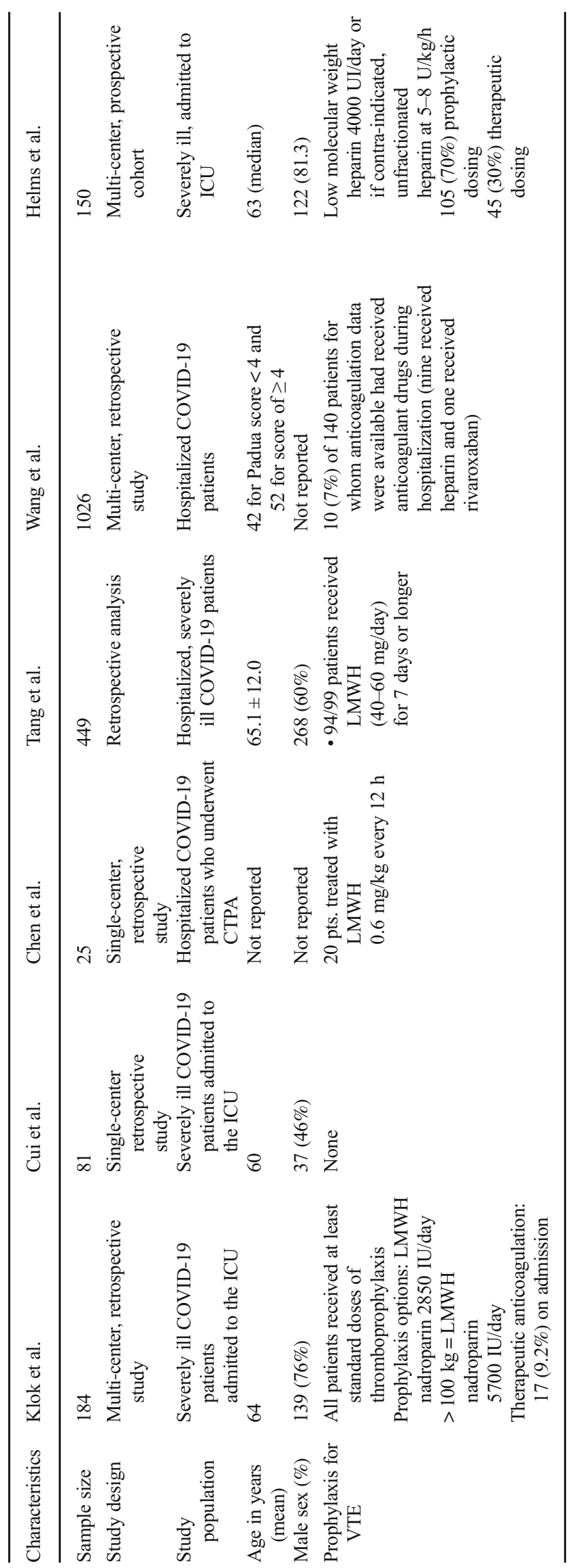


Fig. 1 Proposed algorithm for the evaluation and management of VTE in severe COVID-19 patients. Inflammatory marker refers to C-reactive protein, procalcitonin, ferritin, etc. An example of higher dose VTE prophylaxis includes enoxaparin $0.5 \mathrm{mg} / \mathrm{kg}$ twice daily

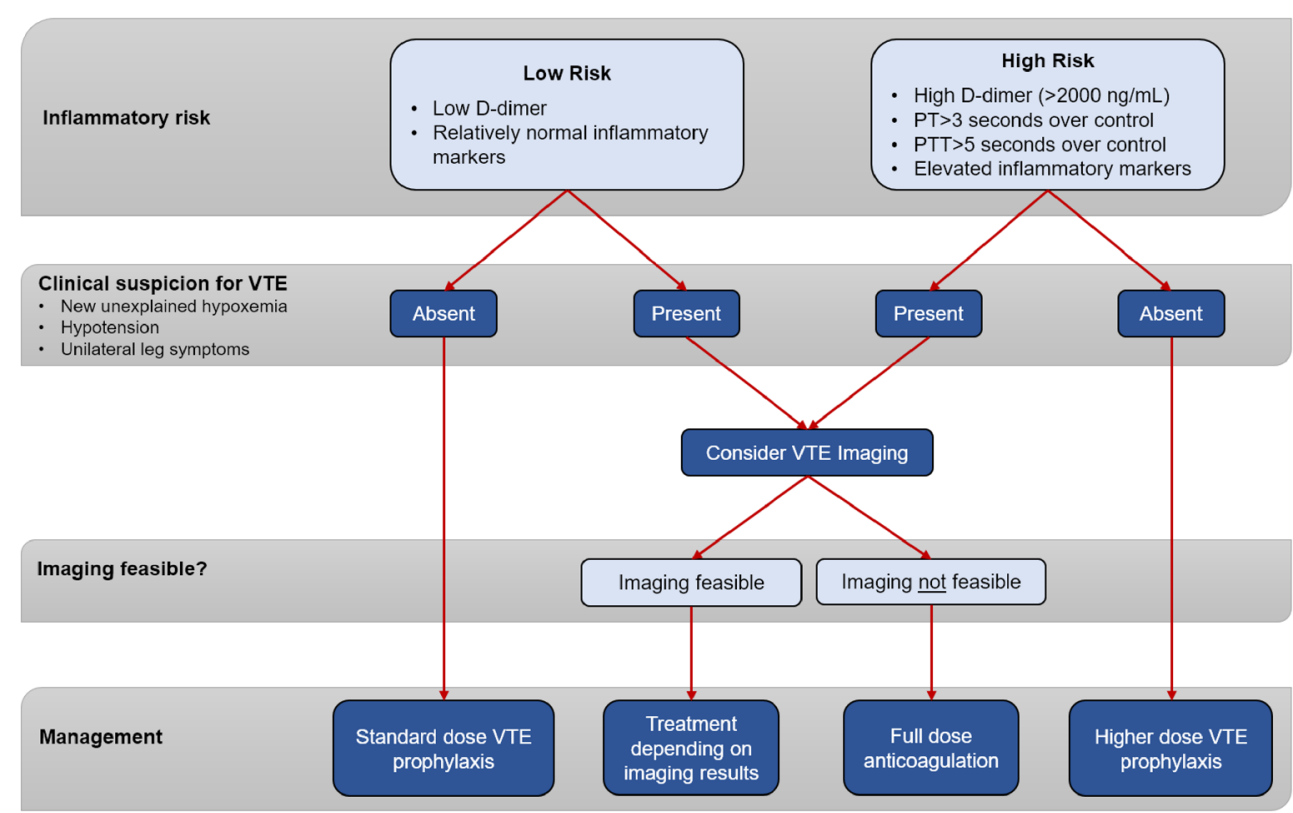

scores were associated with higher risk of bleeding, admission to ICU, intubation, and death due to COVID-19 or complications such as VTE. The study, however, reported significantly lower rates of VTE prophylaxis $(<7 \%)$ [13]. In another retrospective study by Tang et al., the use of low molecular weight heparin (LMWH) treatment was associated with a better 28-day mortality among patients with severe COVID-19 infection and sepsisinduced coagulopathy score $\geq 4$, or D-dimer more than $3 \mu \mathrm{g}$ / $\mathrm{mL}$ (6 times upper limit of normal) [10]. In other studies, strongest association for VTE was found with D-dimer cutoff of $1500 \mathrm{ng} / \mathrm{mL}$ (sensitivity of $85 \%$ and specificity of $89 \%$ ) [3]. However, as noted above, the majority of patients did not receive thromboprophylaxis implying a potentially severe selection bias.

VTE prophylaxis for COVID-19 patients have been incorporated into different guidelines including the Journal of American College of Cardiology state of the art review [14], Swiss consensus statement [15], and NHS model [16]. American Society of Hematology also offers frequently asked questions on VTE on COVID-19 patients. ${ }^{16(\mathrm{p} 19)}$ Until better evidence emerges, we support the Swiss consensus statement that reads: "In patients in intensive care with a large increase in D-dimers, severe inflammation, or signs of hepatic or renal dysfunction or imminent respiratory failure, intermediate or therapeutic dosing of LMWH or unfractionated heparin should be considered, according to the bleeding risk."

\section{Should We Use Extended VTE Prophylaxis (4-6 Weeks) in Severe COVID-19 Patients?}

The risk of VTE among COVID-19 patients that are discharged from the hospital remains unclear with a lack of published data. However, in prior studies, in acutely ill medical patients, extended-duration anticoagulation with rivaroxaban and betrixaban has been shown to reduce the risk of VTE [17]. However, extended anticoagulation may be associated with an increased risk of bleeding [18, 19]. While further studies are needed, extended-duration prophylaxis for 4-6 weeks may be reasonable for some patients such as those with additional risk factors or persistent coagulopathy in the absence of contraindications to anticoagulation therapy.

Acknowledgments MRA, RG, and AK were involved in initial literature search, data collection, and manuscript writing. RP, VRB, and AD drafted the subsequent manuscript. PK is the senior author and subsequently finalized the manuscript for submission. MRA is the primary author.

\section{Compliance with Ethical Standards}

Conflict of Interest The authors declare that they have no conflict of interest.

Human and Animal Rights and Informed Consent This article does not contain any studies with human or animal subjects performed by any of the authors.

\section{References}

1. Obi AT, Tignanelli CJ, Jacobs BN, Arya S, Park PK, Wakefield TW, et al. Empirical systemic anticoagulation is associated with decreased venous thromboembolism in critically ill influenza A H1N1 acute respiratory distress syndrome patients. J Vasc Surg Venous Lymphat Disord. 2019;7(3):317-24. https://doi.org/10. 1016/j.jvsv.2018.08.010.

2. Chen Y-G, Lin T-Y, Huang W-Y, Lin C-L, Dai M-S, Kao C-H. Association between pneumococcal pneumonia and venous thromboembolism in hospitalized patients: a nationwide populationbased study. Respirol Carlton Vic. 2015;20(5):799-804. https:// doi.org/10.1111/resp.12501. 
3. Cui S, Chen S, Li X, Liu S, Wang F. Prevalence of venous thromboembolism in patients with severe novel coronavirus pneumonia. J Thromb Haemost JTH Published online April. 2020;9:1421-4. https://doi.org/10.1111/jth.14830.

4. Klok FA, Kruip MJHA, Meer NJM van der, et al. Incidence of thrombotic complications in critically ill ICU patients with COVID-19. Thromb Res. 2020;0(0). doi:https://doi.org/10.1016/j. thromres.2020.04.013

5. Shi $\mathrm{C}$, Wang $\mathrm{C}$, Wang $\mathrm{H}$, et al. The potential of low molecular weight heparin to mitigate cytokine storm in severe covid-19 patients: a retrospective clinical study. medRxiv. Published online April 7, 2020:2020.03.28.20046144. doi:https://doi.org/10.1101/ 2020.03.28.20046144

6. Campbell CM, Kahwash R. Will complement inhibition be the new target in treating COVID-19 related systemic thrombosis? Circulation Published online April. 2020;9:1739-41. https://doi. org/10.1161/CIRCULATIONAHA.120.047419.

7. Zhang Y, Xiao M, Zhang S, et al. Coagulopathy and antiphospholipid antibodies in patients with Covid-19. $N$ Engl J Med. 2020;0(0):null. doi:https://doi.org/10.1056/NEJMc2007575

8. Chaturvedi S, Braunstein EM, Yuan X, Yu J, Alexander A, Chen H, et al. Complement activity and complement regulatory gene mutations are associated with thrombosis in APS and CAPS. Blood. 2020;135(4):239-51. https://doi.org/10.1182/blood.2019003863.

9. Martin K, Beyer-Westendorf J, Davidson BL, Huisman MV, Sandset PM, Moll S. Use of the direct oral anticoagulants in obese patients: guidance from the SSC of the ISTH. J Thromb Haemost JTH. 2016;14(6):1308-13. https://doi.org/10.1111/jth.13323.

10. Tang N, Bai H, Chen X, Gong J, Li D, Sun Z. Anticoagulant treatment is associated with decreased mortality in severe coronavirus disease 2019 patients with coagulopathy. $J$ Thromb Haemost JTH Published online March. 2020;27:1094-9. https://doi.org/10.1111/ jth.14817.

11. Yin S, Huang M, Li D, Tang N. Difference of coagulation features between severe pneumonia induced by SARS-CoV2 and non-
SARS-CoV2. J Thromb Thrombolysis Published online April. 2020;3. https://doi.org/10.1007/s11239-020-02105-8.

12. Helms J, Tacquard C, Francois Severac. High risk of thrombosis in patients in severe SARS-CoV-2 infection: a multicenter prospective cohort study. doi:https://doi.org/10.1007/s00134-020-06062-x

13. Wang T, Chen R, Liu C, et al. Attention should be paid to venous thromboembolism prophylaxis in the management of COVID-19. Lancet Haematol. 2020;0(0). doi:https://doi.org/10.1016/S23523026(20)30109-5

14. Bikdeli B, Madhavan MV, Jimenez D, Chuich T, Dreyfus I, Driggin E, et al. COVID-19 and thrombotic or thromboembolic disease: implications for prevention, antithrombotic therapy, and followup. J Am Coll Cardiol Published online April. 2020;15. https:// doi.org/10.1016/j.jacc.2020.04.031.

15. Casini A, Alberio L, Angelillo-Scherrer A, Fontana P, Gerber B, Graf L, et al. Thromboprophylaxis and laboratory monitoring for in-hospital patients with Covid-19 - a Swiss consensus statement by the Working Party Hemostasis. Swiss Med Wkly. 2020;150: w20247. https://doi.org/10.4414/smw.2020.20247.

16. Coronavirus. Accessed April 12, 2020. https://www.england.nhs. $\mathrm{uk} /$ coronavirus/

17. Cohen AT, Harrington RA, Goldhaber SZ, Hull RD, Wiens BL, Gold A, et al. Extended thromboprophylaxis with betrixaban in acutely ill medical patients. N Engl J Med. 2016;375(6):534- 44. https://doi.org/10.1056/NEJMoa1601747.

18. COVID-19 and pulmonary embolism - Hematology.org. Accessed April 12, 2020. https:/www.hematology.org:443/covid-19/covid19-and-pulmonary-embolism

19. Cohen AT, Spiro TE, Büller HR, Haskell L, Hu D, Hull R, et al. Rivaroxaban for thromboprophylaxis in acutely ill medical patients. N Engl J Med. 2013;368(6):513-23. https://doi.org/10.1056/ NEJMoa1111096.

Publisher's Note Springer Nature remains neutral with regard to jurisdictional claims in published maps and institutional affiliations. 